\title{
Ultrafast YBCO Photodetector Based on the Kinetic-Inductive Process
}

\author{
Carlo Williams, Ying Xu, Roman Adam, Marian Darula, Oliver Harnack, Jens Scherbel, Michael Siegel, \\ Frank A. Hegmann, and Roman Sobolewski
}

\begin{abstract}
We make use of the nonequilibrium kinetic-inductive effect in epitaxial $\mathrm{YBa}_{2} \mathrm{Cu}_{3} \mathrm{O}_{7-x}(\mathrm{YBCO})$ thin films to demonstrate an ultrafast, high-sensitivity, broadband photodetector. The photoresponse of a 5- $\mu \mathrm{m}$-wide, $10-\mu \mathrm{m}$-long, 100-nm-thick YBCO microbridge embedded in a $20-\mu \mathrm{m}$-wide coplanar strip transmission line was measured using a $>1-\mathrm{THz}$, submillivoltsensitivity electro-optic sampling system. We performed a comprehensive study of the optical power and wavelength dependency on the YBCO photodetector response. The quantum coherence Rothwarf-Taylor model together with the hot-electron relaxation effect were used to explain the measured data and to extract the number of generated quasiparticles for each absorbed photon, revealing the intrinsic quantum yield of our device to be $\sim 450$. The amplitude, response time, and the intrinsic gain of the YBCO photoresponses were observed to be spectrally flat over the tested range of $390 \mathrm{~nm}$ to $810 \mathrm{~nm}$.
\end{abstract}

Index Terms-kinetic inductance, electro-optic sampling, ultrafast photodetectors, YBCO thin film, superconducting optoelectronics.

\section{INTRODUCTION}

$\mathrm{P}$ hotoexcitation investigations of superconducting films by pulsed radiation have been motivated by both the basic physics interest in studies of nonequilibrium dynamics in complex, multibody systems excited by strong perturbations and the practical interest in developing ultrafast and ultrasensitive photodetectors. The experiments toward the former end have led to a clearer picture of the nonequilibrium Cooper-pair and quasiparticle dynamics. Using a subpicosecond electro-optic (EO) sampling system, the single-picosecond electrical response from a current-biased $\mathrm{YBa}_{2} \mathrm{Cu}_{3} \mathrm{O}_{7-x}$ (YBCO) microbridge exposed to femtosecond-pulse optical radiation was observed [1]. In a similar experiment, the intrinsic Cooper-pair-breaking and quasiparticle recombination times were extracted from the photoresponse of a YBCO microbridge current-biased in the superconducting state and maintained at a temperature well below the transition temperature $T_{c}$ [2]. The nonequilibrium quasiparticle dynamics was successfully mod-

Manuscript received September 17, 2000. This work was supported by the US Office of Naval Research Grant No N0014-00-1-0237 and by the National Science Foundation Grant No. INT-0078949.

C. W., Y. X., R. A., and R. S. are with the Department of Electrical and Computer Engineering and Laboratory for Laser Energetics, University of Rochester, Rochester, NY 14627 USA (telephone: 716-275-1808, e-mail: cwilliam@ece.rochester.edu).

R. S. is also with the Institute of Physics, Polish Academy of Sciences, PL-02668, Warsaw, Poland.

M. D., O. H. J. S., and M. S. are with the Institute for Thin Film and Ion Technology, Research Center Jiilich, D-52425, Jülich, Germany.

F. A. H. is with the University of Alberta, Edmonton, Alberta, Canada. eled using either the hot-electron effect (for the response in the resistive state), or the kinetic-inductive photoresponse and the Rothwarf-Taylor (R-T) rate equations in the superconducting state [3]

Refs. [1]-[3] mentioned applications of the YBCO microbridge as an ultrafast photodetector; however, they did not fully address the performance issues, such as quantum yield, that are relevant to an optical detector. In one study [4], an unusual photon resonance effect in $\mathrm{YBCO}$ was reported, suggesting a dramatic wavelength dependence of the YBCO photoresponse. This remains a controversial topic that is yet to be independently verified. In another case, the YBCO response's dependence on the illuminating optical power was discussed [5], but the extreme illumination intensity levels, either low or very high, were not investigated.

We present a comprehensive study of the YBCO microbridge photoresponse signal dependence on the incident illumination optical power and wavelength. A subpicosecond-resolution, submillivolt-voltage-sensitivity EO sampling system was used to measure the voltage responses while varying the input pulse optical power in one case, and the wavelength in another. Our work shows the expected kinetic-inductive photoresponse at low optical intensities, but at higher optical power levels, an additional resistive response is observed. Upon analysis of the kinetic-inductive response using the quantum coherence Rothwarf-Taylor model, the intrinsic gain factor of YBCO is calculated to be $\sim 450$. The wavelength-dependency investigation was conducted at several discrete values in the $390-\mathrm{nm}$ to $810-\mathrm{nm}$ range, and the photoresponse amplitude was found to be spectrally independent.

\section{EXPERIMENTAL SETUP}

Our test structures consisted of several coplanar strip (CPS) transmission lines patterned in a 100-nm-thick YBCO thin film grown on a $10-\mathrm{mm}$ by $10-\mathrm{mm} \mathrm{MgO}$ substrate. The CPS lines were $8 \mathrm{~mm}$ long by $20 \mu \mathrm{m}$ wide and were separated by $10 \mu \mathrm{m}$. Each signal line contained a $5-\mu \mathrm{m}$-wide by $10-\mu \mathrm{m}$-long microbridge at the center. The entire CPS structure, except at the microbridge area, was covered with a 50-nm-thick layer of Au. An optical micrograph of the YBCO CPS structure is shown in Fig. 1. The microbridge was characterized by a zero-resistance transition temperature $T_{c 0}>88 \mathrm{~K}$ and critical current density of $J_{c}>1 \mathrm{MA} / \mathrm{cm}^{2}$ at $77 \mathrm{~K}$.

The measurements were performed using a cryogenic EO sampling system described in detail in [6]. Briefly, the EO sys- 
tem is capable of measuring ultrafast transients with a temporal resolution of $200 \mathrm{fs}$ and a voltage sensitivity of $150 \mu \mathrm{V}$. Fig. 2 shows a schematic configuration of the test structure mounted for the $\mathrm{EO}$ sampling characterization. $\mathrm{ALiTaO}_{3}$ crystal, with a high-reflectivity (HR) coating for infrared light on one side, was clamped onto the sample to facilitate the EO measurements, and wire bonds were used for dc-biasing of the microbridge. The entire sample was mounted on a copper block heat exchanger inside a continuous-flow helium cryostat. Two trains of 100-fs optical pulses from a mode-locked Ti:sapphire laser system provided the EO sampling and the sample excitation beam, respectively. The wavelength of the excitation beam was varied either by frequency-doubling and/or optical parametric techniques. As a result, we were able to conduct our measurements at the following excitation wavelengths: $390 \mathrm{~nm}$, $405 \mathrm{~nm}, 730 \mathrm{~nm}, 750 \mathrm{~nm}$, and $810 \mathrm{~nm}$. The sampling beam's wavelength was always $810 \mathrm{~nm}$. Finally, the $\mathrm{LiTaO}_{3}$ crystal with the HR coating was positioned on the sample in such a

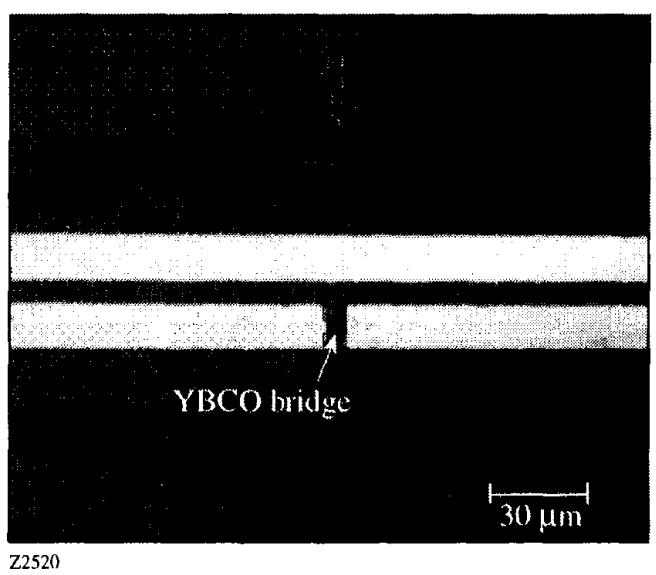

Fig. 1. Optical picture of a YBCO microbridge embedded in a coplanar strip transmission line on $\mathrm{MgO}$ substrate.

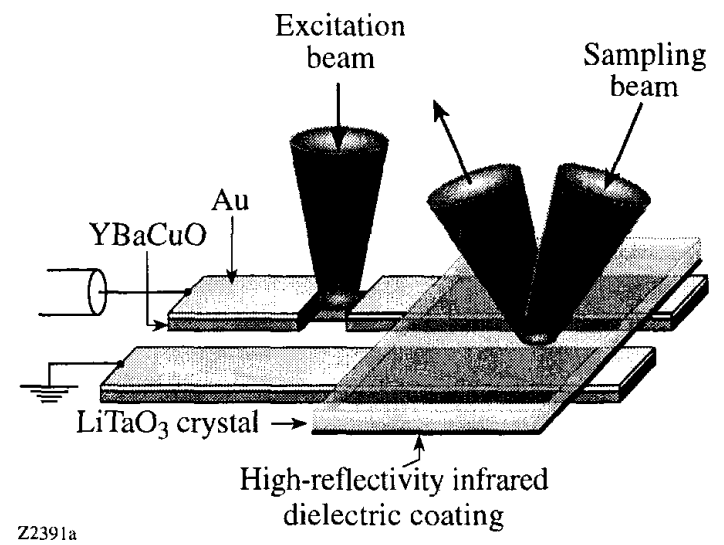

Fig. 2. Schematic representation of the test structure mounted for EO measurements. way that the excitation beam could be delivered to the microbridge area without being reflected or interrupted.

\section{RESULTS AND DISCUSSION \\ A. Optical Input Power Dependence}

To obtain the nonequilibrium kinetic-inductive photoresponse, the microbridge was current-biased in the superconducting state below the critical current $I_{c}$ and at a temperature below $T_{c}$. The open circle waveform in Fig. 3 shows a typically measured photoresponse of the YBCO detector at $60 \mathrm{~K}$ and current-biased at $0.7 I_{c}$; the solid line corresponds to the theoretical fit using the quantum coherence R-T model [7]. The observed bipolar feature of the signal is characteristic for the nonequilibrium kinetic-inductive response [8], while the trailing oscillations are the signature of the quantum coherent interaction between the quasiparticle and the phonon exchange fields.

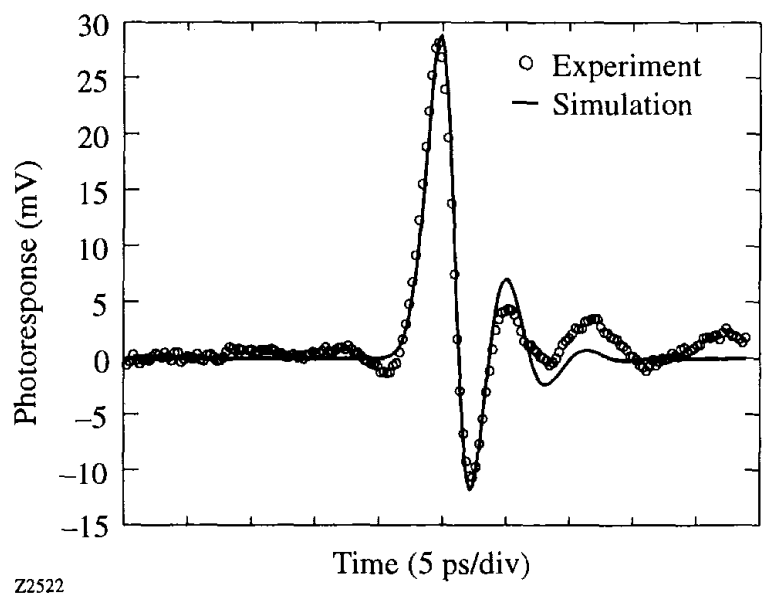

Fig. 3. Measured kinetic-inductive photoresponse of a YBCO photodetector (open circles) and fitted quantum coherent Rothwarf-Taylor model (solid line).

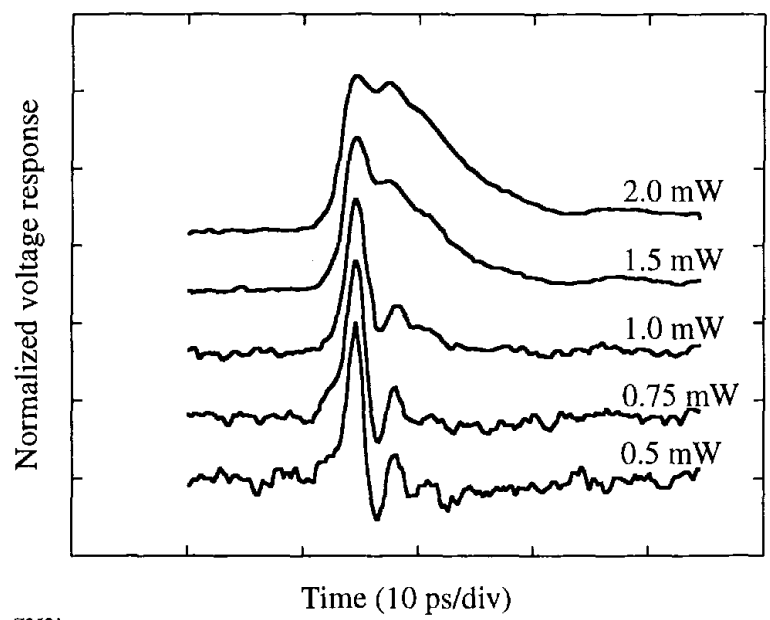

$\mathrm{Z} 2521$

Fig. 4. Sequence of photoresponse waveforms for different incident optical intensities. 
Fig. 4 plots a series of photoresponse waveforms obtained at different levels of the incident optical power. The graph shows a clear evolution of the purely kinetic-inductance response at the optical input power $0.5 \mathrm{~mW}$, to the onset $(0.75 \mathrm{~mW})$ and the development $(1.5 \mathrm{~mW})$ of a second peak and the slow falling edge of the pulse at the increasingly higher optical intensities. Ultimately, the waveform obtained at the 2 -mW illumination power shows a very broad pulse with only minimal influence of the kinetic-inductance effect.

This transformation of the single-picosecond bipolar waveform to a broad photoresponse pulse at higher optical powers was earlier observed by some of us [1], but no satisfactory explanation was provided. Now we demonstrate that it can be explained in terms of a superposition of the kinetic-inductive and the resistive hot-electron photoresponse mechanisms. The quantum coherence $\mathrm{R}-\mathrm{T}$ rate equations assume that the incident optical power is low enough to cause only a small perturbation in the concentration of the quasiparticles [3], [7]. At higher optical intensities, however, such a large number of quasiparticles is generated that the superconducting bridge is pushed into the resistive state. When the $\mathrm{YBCO}$ is currentbiased in the resistive state, a photoresponse can be obtained because of a rise in the electron temperature in the film [9]. It is this hot-electron resistive mechanism that adds to the kinetic-inductive photoresponse at higher optical input powers.

Fig. 5 shows the comparison of the waveform with $1-\mathrm{mW}$ optical power from Fig. 4 to the combined response of the kinetic-inductive response of $0.5-\mathrm{mW}$ optical power and the simulated hot-electron resistive response. This excellent fit between the simulated combined response and the experimental waveform verifies the simultaneous occurrence of the kinetic-inductive and hot-electron photoresponse mechanisms. Similar comparisons (not shown) were also obtained for the waveforms with high optical input powers in Fig. 4.

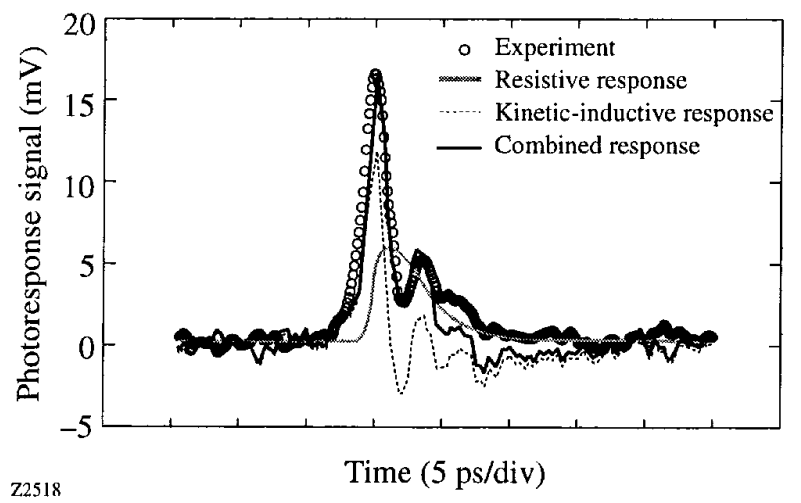

Fig. 5. The kinetic-inductive response with low optical input power is added to the simulation of the hot-electron resistive response to obtain the combined response, which is being compared with the YBCO experimental photoresponse at high optical input power

\section{B. Gain in YBCO Photodetectors}

The calculated gain for the YBCO photodetector is defined as the number of quasiparticles generated due to the absorption of a photon. To calculate the number of photons being absorbed into the superconducting film, we first address the spot size of the input beam relative to the detector area. Throughout the experiment, the incident beam was focused to a $10-\mu \mathrm{m}$-diameter spot size-approximately the dimension of the detector. Because the spatial distribution of the power in the input beam has a Gaussian profile, it is safely assumed that all the optical power was delivered to the microbridge. The actual laser power absorbed $P_{\text {abs }}$ by the detector from the input optical power $P_{\text {in }}$ can be estimated by the following relation:

$$
P_{\mathrm{abs}}=P_{\mathrm{in}} \cdot \eta,
$$

where $\eta$ is the radiation absorption coefficient of a metallic film, given by [10]

$$
\eta=4\left(R_{s} / Z_{0}\right) /\left[\left(R_{s} / Z_{0}\right)\left(n_{\text {sub }}+1\right)+1\right]^{2}
$$

where $n_{\text {sub }}$ is the index of refraction of the MgO substrate, $R_{s}$ is the surface resistance of the YBCO film measured just above $T_{c}$, and $Z_{0}=377 \Omega$ is the free-space impedance. For the $\mathrm{MgO}$ substrate, $n_{\text {sub }}=1.72$ and $R_{s}=800 \Omega$, we obtain an absorption coefficient of $\eta=12 \%$. Thus, the total number of photons per pulse absorbed from $P_{\text {in }}=1.5 \mathrm{~mW}$ at $400-\mathrm{nm}$ wavelength was $1 \times 10^{18} \mathrm{~cm}^{-3}$.

The number of quasiparticles generated can be explained within the simple model of relaxation processes in a thin superconducting film [11]. In the case of our YBCO photodetector, the actual number of quasiparticles generated was obtained by successfully fitting the R-T equations to our experimental data. The optical power absorbed into the superconductor that instigates the quasiparticle-generation process is described within the R-T model as

$$
i_{(\mathrm{QP})}(t)=A \exp \left(-t^{2} / \tau_{T}\right)
$$

where $A$ is the optical intensity parameter and $\tau_{T}$ is the timeconstant parameter that describes the thermalization time of the optical energy within the quasiparticle. It is the parameter $A$ that is extracted from the R-T fit to the experimental data and is used as the factor to calculate the total number of photogenerated quasiparticles:

$$
N_{\mathrm{QP}-\text { gen }}=A \cdot N_{\mathrm{tot}}\left[\frac{T}{T_{c}}\right]^{2},
$$

where $N_{\text {tot }}=2.16 \times 10^{21} \mathrm{~cm}^{-3}$ is the density of electrons in the material. Thus, for the photoresponse in Fig. 3 where $1.5-\mathrm{mW}$ 
average power of 400-nm-wavelength light pulses was delivered to the detector, a value of $A=0.45$ was extracted, $N_{\mathrm{QP} \text {-gen }}$ then equals $4.5 \times 10^{20} \mathrm{~cm}^{-3}$ at $T=60 \mathrm{~K}$ and the intrinsic gain factor of the YBCO detector is calculated to be 450 .

\section{Wavelength Dependence}

Fig. 6 shows a series of waveforms acquired from an excitation wavelength of $390,405,730,750$, and $810 \mathrm{~nm}$. The waveforms were taken under similar experimental conditions. Contrary to reports that there might be a spectral dependence on the nonequilibrium kinetic-inductive effect [4], no such dependence was observed in our case. This result supports our interpretation that in optimally doped YBCO films, optical absorption is due to the free-carrier absorption mechanism and manifests itself as kinetic-inductive and hot-electron resistive photoresponse.

\section{CONCLUSION}

A comprehensive study of the kinetic-inductive photoresponse of YBCO toward an optical detector was conducted. An EO sampler was used to record the signal from a YBCO microbridge while varying the optical input power to extract a full-power and wavelength dependency. The photoresponse amplitude and waveform were consistent with the theoretical kinetic-inductive model at low power levels, but at higher power levels the observed photoresponse was a combination of the kinetic-inductive and the hot-electron resistive mechanisms. The photoresponse amplitude was ob-

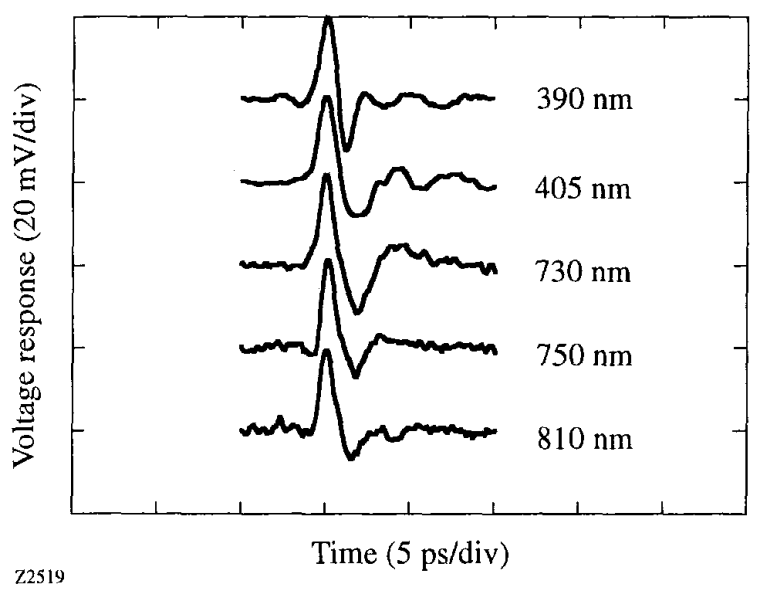

Fig. 6. Series of waveforms acquired with different excitation wavelengths under similar conditions. served to be spectrally independent, and the gain of the YBCO detector was calculated to be $\sim 450$. Thus, the YBCO detector is shown to be a high-gain, broadband, and ultrafast optical detector.

\section{ACKNOWLEDGMENT}

R. Adam and Y. Xu acknowledge support from the Frank Horton Graduate Fellowship Program.

\section{REFERENCES}

[1] F. A. Hegmann, D. Jacobs-Perkins, C.-C. Wang, S. H. Moffat, R. A. Hughes, J. S. Preston, M. Currie, P. M. Fauchet, T. Y. Hsiang, and R. Sobolewski, "Electro-optic sampling of 1.5-ps photoresponse signal from $\mathrm{YBa}_{2} \mathrm{Cu}_{3} \mathrm{O}_{7 . \delta}$ thin films," Appl. Phys. Lett., vol. 67, pp. 285-287, 1995.

[2] M. Lindgren, M. Currie, C. Williams, T. Y. Hsiang, P. M. Fauchet, R. Sobolewski, S. H. Moffat, R. A. Hughes, J. S. Preston, and F. A. Hegmann, "Intrinsic picosecond response times of Y-Ba-Cu-O superconducting photodetectors," Appl. Phys. Lett., vol. 74, pp. 853-855, 1999.

[3] R. Sobolewski, "Ultrafast dynamics of nonequilibrium quasi-particle in high-temperature superconductors," in Superconducting and Related Oxides: Physics and Nanoengineering III, D. Pavuna and I. Bozovic, Eds. Bellingham, WA: SPIE, 1998, Vol. 3481, pp. 480-491.

[4] Y. G. Zhao, W. L. Cao, J. J. Li, H. D. Drew, R. Shreekala, C. H. Lee, S. P. Pai, M. Rajeswari, S. B. Ogale, R. P. Sharma, G. Baskaran, and T. Venkatesan, "Unusual photon energy dependence of the Cooper pair breaking rate in $\mathrm{YBa}_{2} \mathrm{Cu}_{3} \mathrm{O}_{7-\delta}$ epitaxial thin films," J. Supercond., vol. 12, pp. 675-680, 1999.

[5] C. Williams, R. Adam, Q. Xie, R. Sobolewski, and O. Harnack, "Nonequilibrium kinetic inductive response of $\mathrm{Y}-\mathrm{Ba}-\mathrm{Cu}-\mathrm{O}$ photodetectors," Supercond. Sci. Technol., vol. 12, pp. 843-846, 1999.

[6] M. Lindgren, M. Currie, C. A. Williams, T. Y. Hsiang, P. M. Fauchet, R. Sobolewski, S. H. Moffat, R. A. Hughes, J. S. Preston, and F. A. Hegmann, "Ultrafast photoresponse in microbridges and pulse propagation in transmission lines made from high- $T_{c}$ superconducting Y-Ba-Cu-O thin films," IEEE J. Sel. Top. Quantum Electron., vol. 2, pp. 668-678, 1996.

[7] R. Sobolewski, Y. Xu, C. Williams, and J. Mostowski, "Analysis of the nonequilibrium kinetic-inductive photoresponse of YBCO thin films by use of the quantum coherence model," to be published.

[8] N. Bluzer, "Temporal relaxation of nonequilibrium in $\mathrm{Y}-\mathrm{Ba}-\mathrm{Cu}-\mathrm{O}$ measured from transient photoimpedance response," Phys. Rev. B, vol. 44, pp. 10222-10223, 1991.

[9] A. D. Semenov, R. S. Nebosis, Yu. P. Gousev, M. A. Heusinger, and K. F. Renk, "Analysis of the nonequilibrium photoresponse of superconducting films to pulsed radiation by use of a two-temperature model," Phys. Rev. B, vol. 52, pp. 581-590, 1995.

[10] M. Born and E. Wolf, Principles of Optics: Electromagnetic Theory of Propagation, Interference, and Diffraction of Light, 7th expanded ed. Cambridge: Cambridge University Press, 1999, pp. 752-758.

[11] K. S. Il'in, I. I. Milostnaya, A. A. Verevkin, G. N. Gol'tsman, E. M. Gershenzon, and R. Sobolewski, "Ultimate quantum efficiency of a superconducting hot-electron photodetector," Appl. Phys. Lett., vol. 73, pp. 3938-3940, 1998. 